\title{
Study of the Effect of Gamma Irradiation on the Mechanical Properties of Polyvinyl Alcohol Based Gelatin Blend Film
}

\author{
Kamol Dey ${ }^{*}$, Sumon Ganguli1, Sushanta Ghoshal2, Mubarak A. Khan2, Ruhul A. Khan² \\ ${ }^{1}$ Department of Applied \& Environmental Chemistry, Faculty of Science, University of Chittagong, Chittagong, \\ Bangladesh \\ ${ }^{2}$ Institute of Radiation and Polymer Technology, Bangladesh Atomic Energy Commission, Dhaka, Bangladesh \\ Email: ${ }^{*}$ kamolacct@gmail.com
}

Received 9 May 2014; revised 23 June 2014; accepted 5 August 2014

Copyright (C) 2014 by authors and OALib.

This work is licensed under the Creative Commons Attribution International License (CC BY).

http://creativecommons.org/licenses/by/4.0/

(c) (i) Open Access

\section{Abstract}

Blend film of polyvinyl alcohol (PVA) based gelatin was prepared by mixing aqueous solutions of both samples. The mixed aqueous solutions of both samples in different ratios (P1, P2, P3, P4 and P5) were then cast onto silicon paper covered glass plate to form film. These films were then irradiated under gamma radiation $\left({ }^{60} \mathrm{Co}\right.$ ) with different intensities (total dose) like 50, 100, 150, 250 and $500 \mathrm{krad}$ to investigate the irradiation effect on the mechanical properties of these films. It was found that, due to incorporating radiation, the tensile strength (TS) and elongation at break (Eb) were improved up to some radiation doses and then again decreased. The highest TS (32 MPa) for blends was observed for $5 \%$ gelatin containing PVA film at $50 \mathrm{krad}$ which was $19 \%$ higher than that of non-irradiated blend and the highest Eb (165\%) was found for $10 \%$ gelatin containing PVA film at $100 \mathrm{krad}$. FTIR spectra of the pure and blend films were taken for structural characterization. Morphological investigation of the films was investigated by Scanning Electron Microscopy (SEM) analysis.

\section{Keywords}

Gelatin, Polyvinyl Alcohol, Blending, Cross-Linking, Gamma Radiation

Subject Areas: Composite Material, High Polymer Chemistry

\section{Introduction}

Polymer blending is one of the effective methods for providing new desirable polymeric materials for a variety

${ }^{*}$ Corresponding author.

How to cite this paper: Dey, K., Ganguli, S., Ghoshal, S., Khan, M.A. and Khan, R.A. (2014) Study of the Effect of Gamma Irradiation on the Mechanical Properties of Polyvinyl Alcohol Based Gelatin Blend Film. Open Access Library Journal, 1: e639. http://dx.doi.org/10.4236/oalib.1100639 
of applications. Therefore, polymer blends have attracted increasing interest in both industrial and scientific fields. An important property of polymer blend is the miscibility of its components because it affects the mechanical properties, the morphology, and the permeability and degradation. The blending of two or more polymers has become an increasingly important technique for improving the cost-performance ratio of commercial polymer plastics. For example, blending may be used to reduce the cost of an expensive engineering thermoplastic, to improve the process ability of a high temperature or heat sensitive thermoplastic, or to improve impact resistant. Bio-artificial polymeric materials have been prepared using polyvinyl alcohol (PVA), as synthetic components and gelatin as biological components. PVA is a hydrolysis product of poly-(vinyl acetate) (PVAc) and is a polar, water soluble, synthetic polymer. Besides, it is recognized as one of the few synthetic polymers truly biodegradable under both aerobic and anaerobic conditions. PVA has excellent film forming, emulsifying, and adhesive properties. It is also resistant to oil, grease and solvent. It is odorless and nontoxic. It has high tensile strength and flexibility, as well as high oxygen and aroma barrier properties. PVA is an atactic material but exhibits crystallinity as the hydroxyl groups are small enough to fit into the lattice without disrupting it [1]-[3].

Gelatin is widely found in nature and is the major constituent of skin, bones and connective tissue. Gelatin can easily be obtained by a controlled hydrolysis of the fibrous insoluble protein, collagen. Gelatin is a structural protein and normally contains about $15 \%$ water, $1 \%$ - $4 \%$ inorganic salts and trace amount of grease. The properties of gelatin depend on the major protein constituent derived from the breakdown of collagen. Elemental percentages of gelatin are as follows: Carbon 50.5\%, Hydrogen 6.8\%, Nitrogen $17 \%$ and Oxygen $25.2 \%$. Its most frequent uses in the biomedical field include hard and soft capsules, microspheres, wound dressing and three-dimensional tissue regeneration. Gelatin produces flexible tough films when cast together with glycerin or sorbitol. Many attempts, such as physical and chemical treatments, lead to changes in the surface structure and surface energy of the films [4]-[9].

Radiation treatment can introduce better surface cross-linking between natural and synthetic polymers, and thus reduce the hydrophilic nature of the polymeric film. Research has been previously done to explore the irradiation effects on gelatin. It is reported that, gamma radiation increased the cross-linking between protein chains which increase the mechanical properties of the film. It is observed that gamma radiation enhance the tensile properties of biopolymeric films [10]-[15]. In the present study, PVA based gelatin blend films with different composition were prepared to investigate their mechanical and structural properties. The effect of gamma irradiation on the mechanical properties of the films was also investigated.

\section{Experimental}

\subsection{Materials}

Gelatin (bloom strength 185; pharmaceutical grade) was collected from the Opsonin Pharma Limited, Barishal, Bangladesh. The synthetic polymer PVA (Molecular Weight: 72,000) was purchased from Fluka Chemie AGCH9470 Buchs.

\subsection{Methods}

\subsubsection{Preparation of Films}

Gelatin was dissolved in hot water to form solution. PVA was also dissolved in hot water with constant stirring. Then a calculated amount of gelatin solution (5\%, 10\%, 15\% and 20\%) was mixed with the PVA solution. PVA containing $5 \%, 10 \%, 15 \%$ and $20 \%$ gelatin solution was blended for preparing films. The solution was blended in hot water for about one and half an hour to produce homogeneous solution. The solution was then cast onto the silicon paper covered glass plate to form film. The solution was maintained into a thickness of $4 \mathrm{~mm}$ on the glass plate. The solution was dried into films at room temperature for 48 hours. The dried films were then peeled from the silicon cloth and cut with a conventional scissor into small pieces of length $70 \mathrm{~mm}$ and width $10 \mathrm{~mm}$. The thickness of the dried film was about $0.30 \mathrm{~mm}$.

\subsubsection{Treatment of Films}

PVA and PVA/gelatin blend films were irradiated under gamma radiation (Co-60) with different intensities (total dose) like 50, 100, 150, 250 and $500 \mathrm{krad}$. The films were then subjected to various characterizations. 


\subsubsection{Mechanical Properties of Films}

Tensile strength (TS) and elongation at break (Eb) of the films were investigated by the Universal Testing Machine (INSTRON, model 1011, UK). The load range was $500 \mathrm{~N}$ with $10 \mathrm{~mm} / \mathrm{min}$ crosshead speed and $30 \mathrm{~mm}$ gauge length. The dimensions of the test specimen were $70 \mathrm{~mm} \times 10 \mathrm{~mm} \times 0.30 \mathrm{~mm}$.

\subsubsection{Fourier Transform Infra-Red (FTIR) Spectroscopy}

Pure PVA film, pure gelatin film and a blend (95\% PVA + 5\% gelatin) were characterized by FTIR analysis. The FTIR spectra of the films were recorded using a Spectrum One spectrophotometer (Perkin-Elmer, Woodbridge, ON, Canada) equipped with an attenuated total reflectance (ATR) device for solids analysis and a high linearity lithium tantalate (HLLT) detector. Spectra were analyzed using the Spectrum 6.3 .5 software. Films were stored at room temperature for 72 hour in a desiccator containing saturated $\mathrm{NaBr}$ solution to ensure a stabilized atmosphere of $60 \% \mathrm{RH}$ at $20^{\circ} \mathrm{C}$. Films were then placed onto a zinc selenide crystal, and the analysis was performed within the spectral region of 400 to $2400 \mathrm{~cm}^{-1}$ with 64 scans recorded at a $4 \mathrm{~cm}^{-1}$ resolution. After attenuation of total reflectance and baseline correction, spectra were normalized with a limit ordinate of 1.5 absorbance units. Resulting FTIR spectra were compared in order to evaluate the effects of radiation on PVA and gelatin blended films.

\subsubsection{Scanning Electron Microscopy (SEM)}

The surface morphology of PVA, gelatin and their blends was studied using a JEOL 6400 SEM at an accelerating voltage of $10 \mathrm{keV}$. SEM specimens were sputter-coated with gold.

\section{Results and Discussion}

\subsection{Mechanical Properties of the Untreated and Treated Films}

Four different blends (P2, P3, P4 and P5) were prepared from various concentrations of PVA/gelatin solutions that were shown in Table 1 . When blends were prepared by mixing PVA and gelatin water solution, a homogeneous water solution was produced, thus showing compatibility of the two components in the solvent, whereas cast films appeared homogeneous only for a limited amount (20\%) of gelatin content. Thus, in blends with the higher amount of $20 \%$ gelatin, phase separation and opacity were evident. These were impossible to handle. So we investigated the mechanical properties of the films containing $0 \%, 5 \%, 10 \%, 15 \%$ and $20 \%$ (P1, P2, P3, P4 and P5) gelatin. The TS is the most important mechanical property for many application of polymer [16]. The results of TS values of the not irradiated films for P1, P2, P3, P4, and P5 formulation were plotted in Figure 1. It was observed that with the loading of gelatin the TS of the base polymer (PVA) were significantly decreased. The TS values of non irradiated and irradiated films were plotted in Figure 2 against gamma irradiation dose. It was found that due to incorporating gamma irradiation the TS values were improved up to some radiation dose and then again decreased for both pure PVA (P1) and blend film (P2, P3, P4 and P5) respectively. In case of pure PVA film, TS values were increased with the increase of irradiation doses and TS value attained maximum of 36 MPa at $150 \mathrm{krad}$ and then decreased with increasing radiation intensities. Cross-linking and chain scission occurred when polymers are exposed to gamma radiation [17]-[20]. Polysaccharides and other natural polymers generally degrade by breaking the glycosidic linkage under gamma radiation [18]. Gelatin is a natural biopolymer which consists of protein molecules and can easily fragment due to gamma radiation. The exposure of

Table 1. Composition of different blending formulation (\% w/w).

\begin{tabular}{ccc}
\hline & Composition $(\% \mathrm{w} / \mathrm{w})$ & \\
\hline Formulation & PVA (\%) & Gelatin (\%) \\
\hline P1 & 100 & 00 \\
P2 & 95 & 10 \\
P3 & 90 & 15 \\
P4 & 85 & 20 \\
P5 & 80 & 20 \\
\hline
\end{tabular}




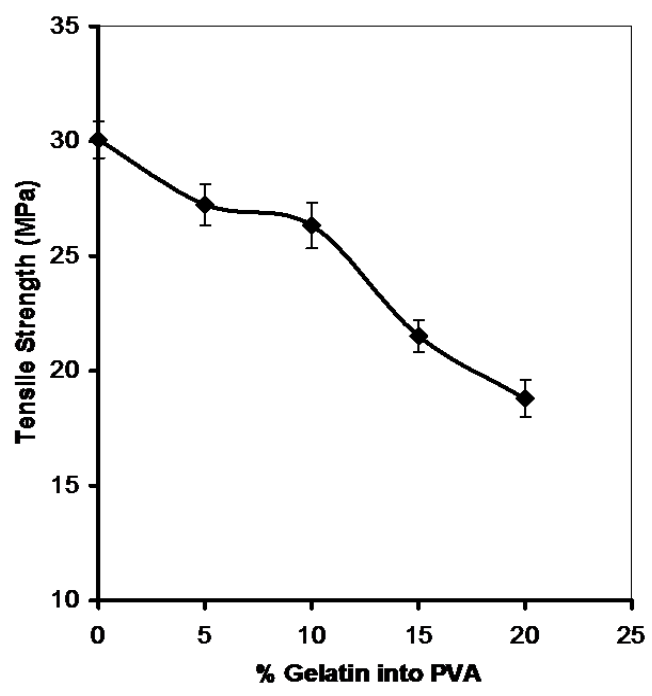

Figure 1. Tensile strength (TS) of gelatin containing PVA based film.

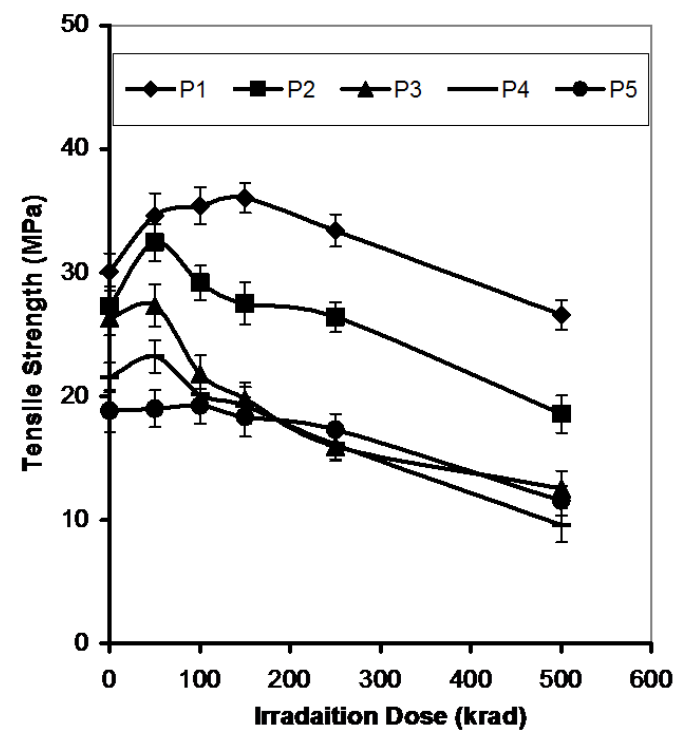

Figure 2. Tensile strength (TS) of PVA based gelatin film against different gamma irradiation dose (krad).

gamma radiation produces the free radicals due to the chain scission of the gelatin molecules. The generated free radicals may crosslink each other and form more crosslinked sites with the exposure of gamma radiation; as a result, mechanical properties might be increased. Higher gamma radiation does might have caused degradation of the polymer and the film became hard and brittle whilst at lower doses cross-linking might have dominated over chain seasoning.

From the Figure 2, in case of irradiated blend film, it was also evident that the TS values were reached a maximum up to approximately $32 \mathrm{MPa}$ at $50 \mathrm{krad}$ for P2 blend film and then decreased for further increasing of radiation intensity as well as PVA/gelatin concentration. Figure 3 showed the tensile strength (TS) change with gamma irradiation dose at 50 krad. From the Figure 3, it was clear that TS value of gamma treated film was higher than that of untreated film. When the PVA/gelatin film subjected to the radiation, hydroxyl group from PVA and gelatin radicals were initiated to form cross linked network. So, TS value increased with radiation, but higher radiation doses caused degradation due to the breaking of the polymer chains. However, the present samples prepared by blending of PVA and gelatin might not be able to successfully cross-linked but instead were degraded easily by an irradiation of a higher dose than 50 krad. So, at higher radiation doses TS value decreased. 
Elongation is an important mechanical property in the application of polymer. The results of Eb of the non irradiated films for P1, P2, P3, P4 and P5 formulation were shown in Figure 4. It was observed that, Eb was decreased drastically due to incorporating gelatin into the PVA film. It was attributed because of the gradually decreased in concentration of highly flexible PVA in to the blend film. The Eb values of irradiated films were plotted in Figure 5 against gamma radiation dose. It was found that for both virgin PVA and blend film Eb was increased up to some radiation dose. But after reaching to a maximum limit, Eb was decreased with the increase of radiation doses. This was due to the radiation degradation of the film with increasing radiation intensities.

In case of irradiated pure PVA film (P1) the maximum value of Eb was found $174 \%$ at $100 \mathrm{krad}$, and then decreased for further increasing of radiation intensity. In case of irradiated blend film, the highest Eb was found 165\% for 10\% gelatin containing PVA blend (P3 formulation) at $100 \mathrm{krad}$. At 150, 250 and $500 \mathrm{krad}$, the Eb values were $142 \%, 71 \%$ and $60 \%$, respectively. The formulation of P1, P2, P4 and P5 also showed the same trend like P3 formulation. Elongation at break (Eb) change with gamma irradiation dose at 100 krad was shown in Figure 6. From the Figure 6, it was clear that Eb value of gamma treated film was higher than that of untreated film.

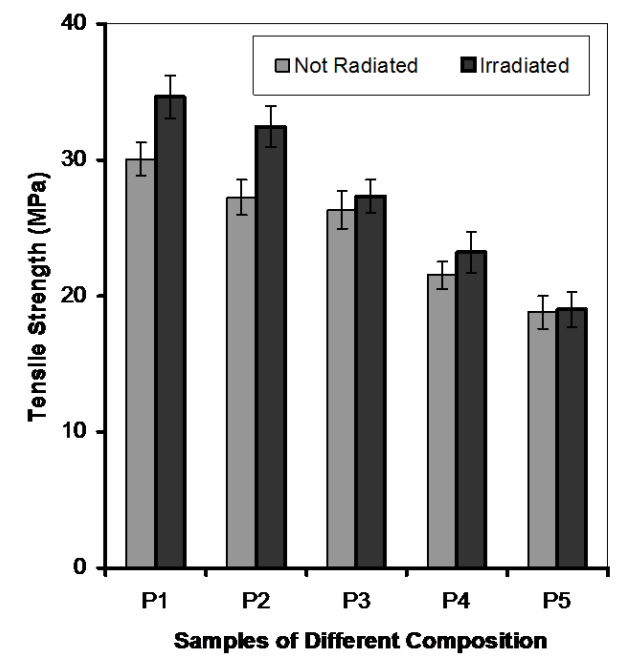

Figure 3. Tensile strength (TS) change with gamma irradiation dose at 50 krad.

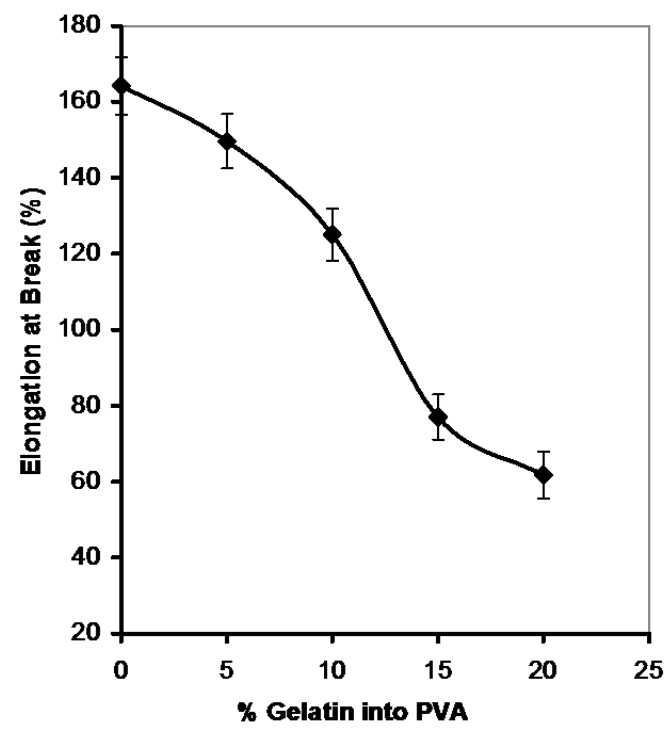

Figure 4. Elongation at break (Eb) of gelatin containing PVA based film. 


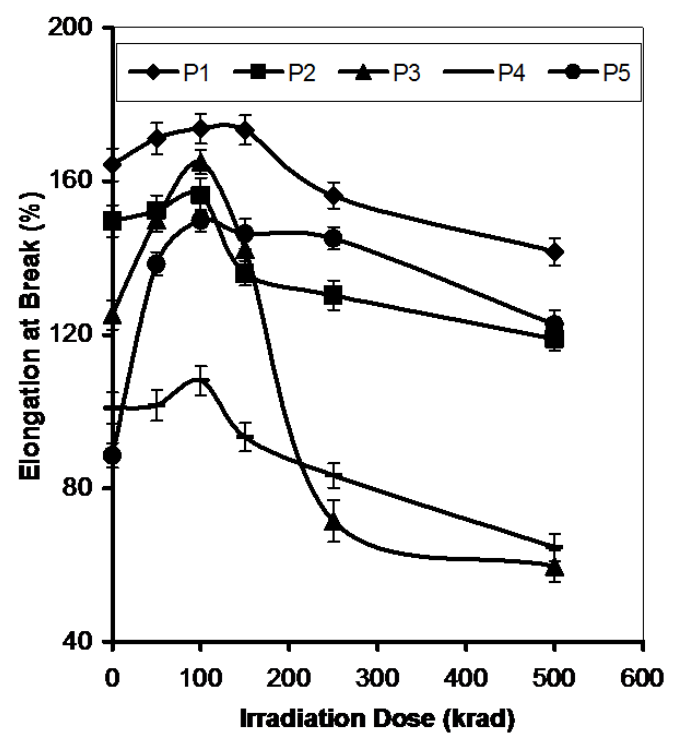

Figure 5. Elongation at break (Eb) of PVA based gelatin film against different gamma irradiation dose (krad).

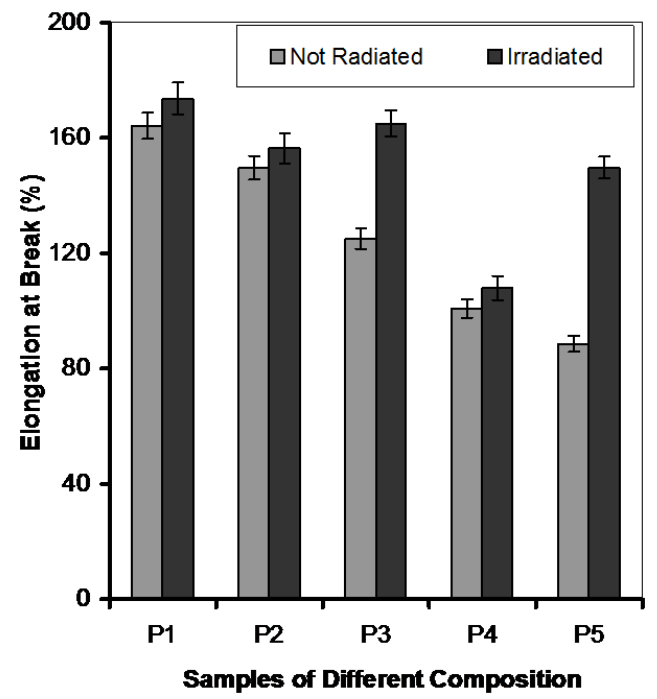

Figure 6. Elongation at break (Eb) change with gamma irradiation dose at 100 krad.

\subsection{Fourier Transformed Infrared Spectroscopy (FTIR) Analysis}

FTIR is of importance to study the molecular structure. The width and intensity of spectral bands as well as position of peaks are all sensitive to environmental changes and to conformations of macromolecule on molecular level. Intermolecular interactions occur when two polymers are compatible. So the FTIR spectra of the blends are different from those of the pure polymer, which is advantageous to study the extent of compatibility of the blend polymers. FTIR spectrophotometer has also been found to be a valuable tool in studying graft copolymerization reactions. Figures 7-9 showed the infrared spectra for the films of gamma untreated pure PVA, pure gelatin and the gamma irradiated P2 formulation (95\% PVA + 5\% gelatin) in the wave number range of $4000-400$ $\mathrm{cm}^{-1}$. From the Figure 7, the FTIR spectra of PVA showed a broad peak around $3000-3600 \mathrm{~cm}^{-1}$ may be due to stretching of hydroxyl groups (-OH) and C-H stretching. The peak at $1088 \mathrm{~cm}^{-1}$ indicated the C-O stretch of secondary alcoholic groups. The FTIR spectra of gelatin; Figure 8 showed peaks at $3300-3600 \mathrm{~cm}^{-1}$ due to -NH stretching of secondary amide and also show C-H stretching at $2922 \mathrm{~cm}^{-1}$. The most distinctive spectral 


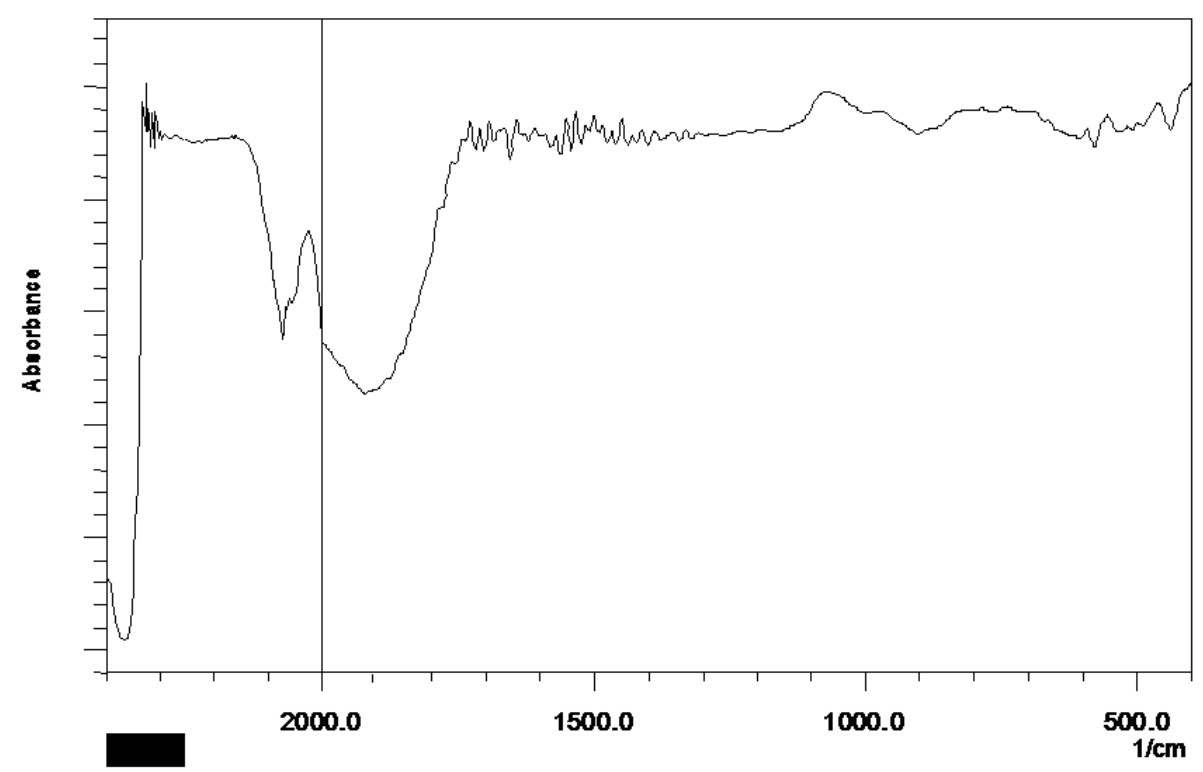

Figure 7. FTIR spectrum of gamma untreated pure PVA.

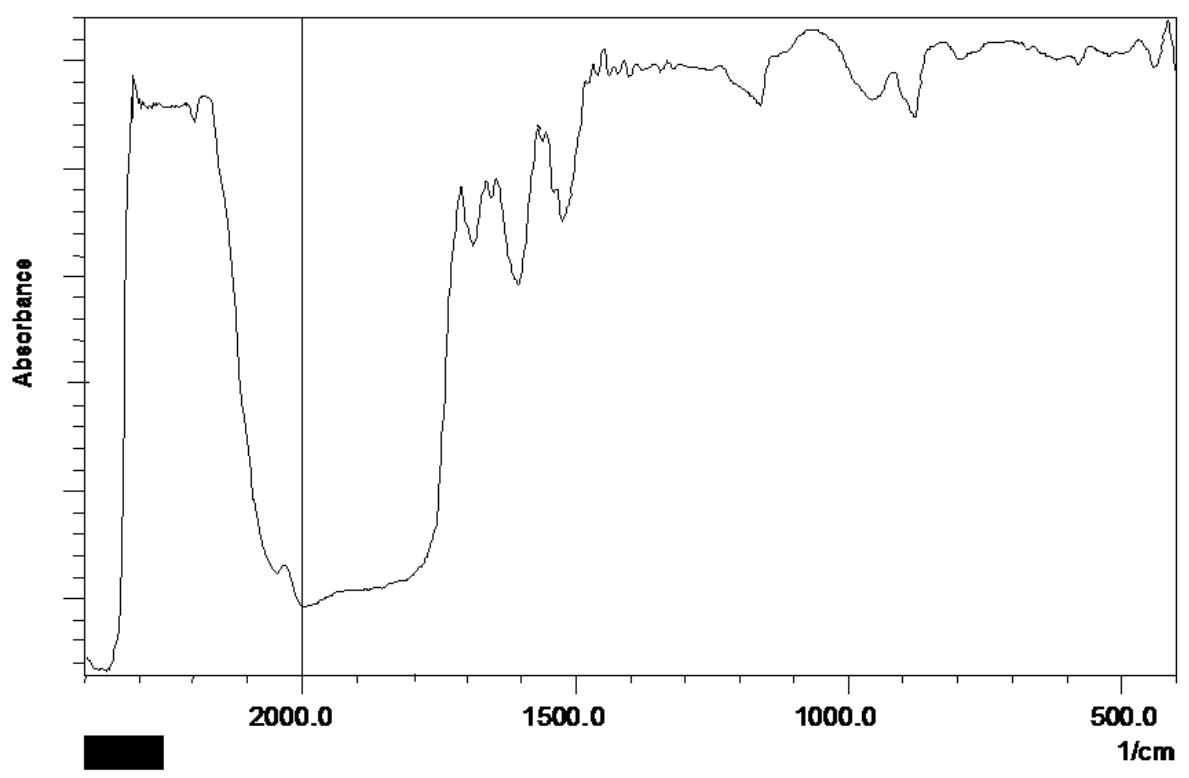

Figure 8. FTIR spectrum of gamma untreated pure gelatin.

features for the protein were the strong amide I and II bands centered at approximately at 1640 and $1550 \mathrm{~cm}^{-1}$, respectively. The amide I absorption was primarily due to the stretching vibration of the $\mathrm{C}=\mathrm{O}$ bond and the amide II band was due to the coupling of the bending of the - $\mathrm{NH}$ bond and the stretching of the C-N bond. The FTIR spectrum of gamma irradiated P2 formulation was given in Figure 9. The spectra of the blend showed a peak around $3500 \mathrm{~cm}^{-1}$, indicating the presence of a hydroxyl group with polymeric association and a secondary amide. The grafted product did not show any characteristic peak corresponding to carbonyl group and amino group indicating the cross-linking through these groups.

\subsection{Scanning Electron Microscopic (SEM) Analysis}

In order to study surface morphology SEM study was undertaken. Scanning electron microscopic (SEM) image of untreated pure PVA, pure gelatin and an irradiated blend of P2 formulation were shown in Figures 10-12. The 


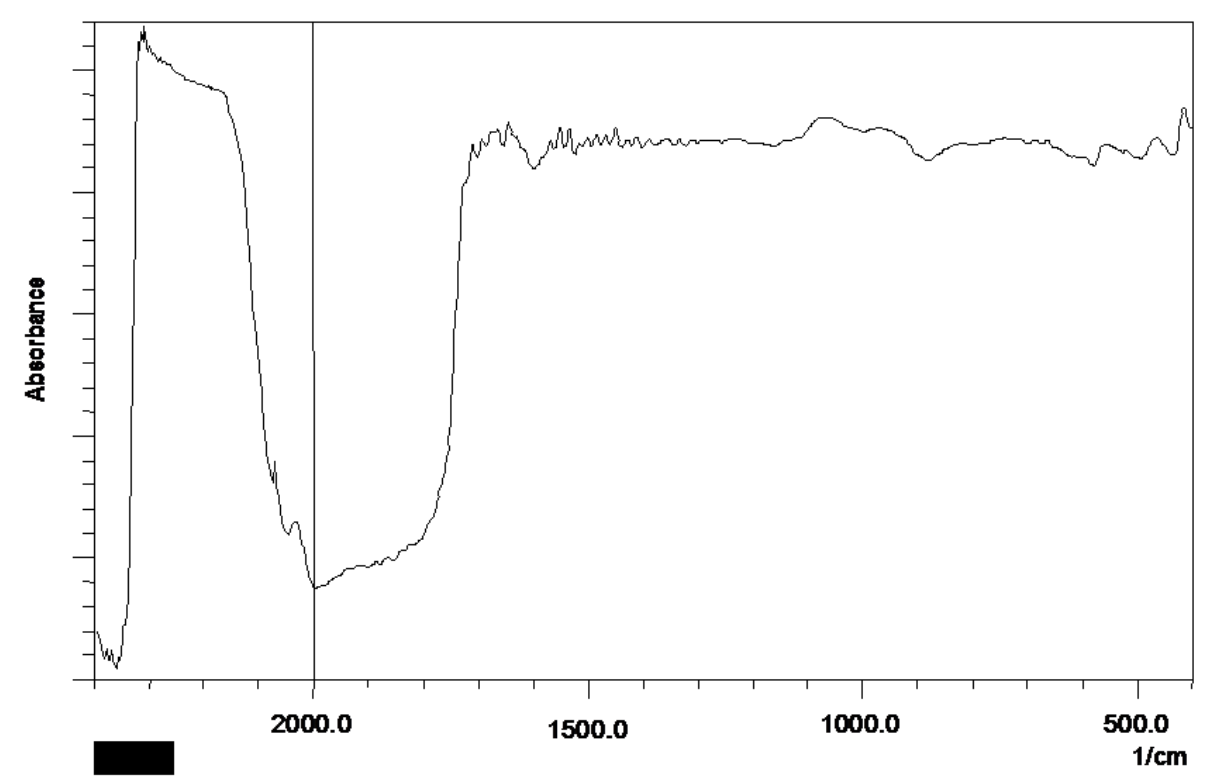

Figure 9. FTIR spectrum of gamma irradiated P2 formulation (95\% PVA + 5\% gelatin).

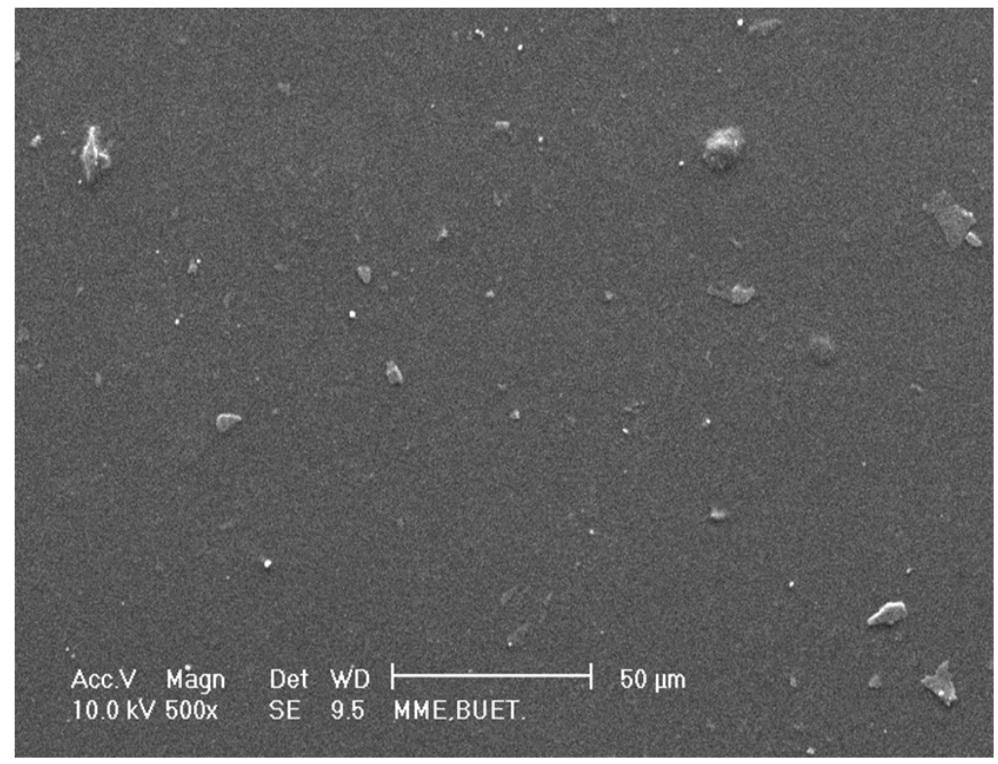

Figure 10. SEM image of gamma untreated pure PVA film.

SEM of PVA showed better film forming property than gelatin. In Figure 11, some unbound micro granules were observed. From the Figure 12, it was evident that some type of interaction did occur between gelatin and PVA due to gamma irradiation.

\section{Conclusion}

Polyvinyl alcohol (PVA) and gelatin-based films were prepared by solvent casting. Then films were irradiated under gamma irradiation (50 to $500 \mathrm{krad}$ ) to investigate the effect of irradiation on the mechanical properties of the prepared films. The highest tensile strength (TS) and elongation at break (Eb) were found to be $27 \mathrm{MPa}$ and $150 \%$ respectively with the formulation P2 (95\% PVA + 5\% gelatin). It was found that, due to the exposure of radiation, the TS and Eb were improved up to the certain doses of radiation then decreased. The highest TS for irradiated blend films was found $32 \mathrm{MPa}$ at $50 \mathrm{krad}$ (P2 formulation) which was 19\% higher than that of the 


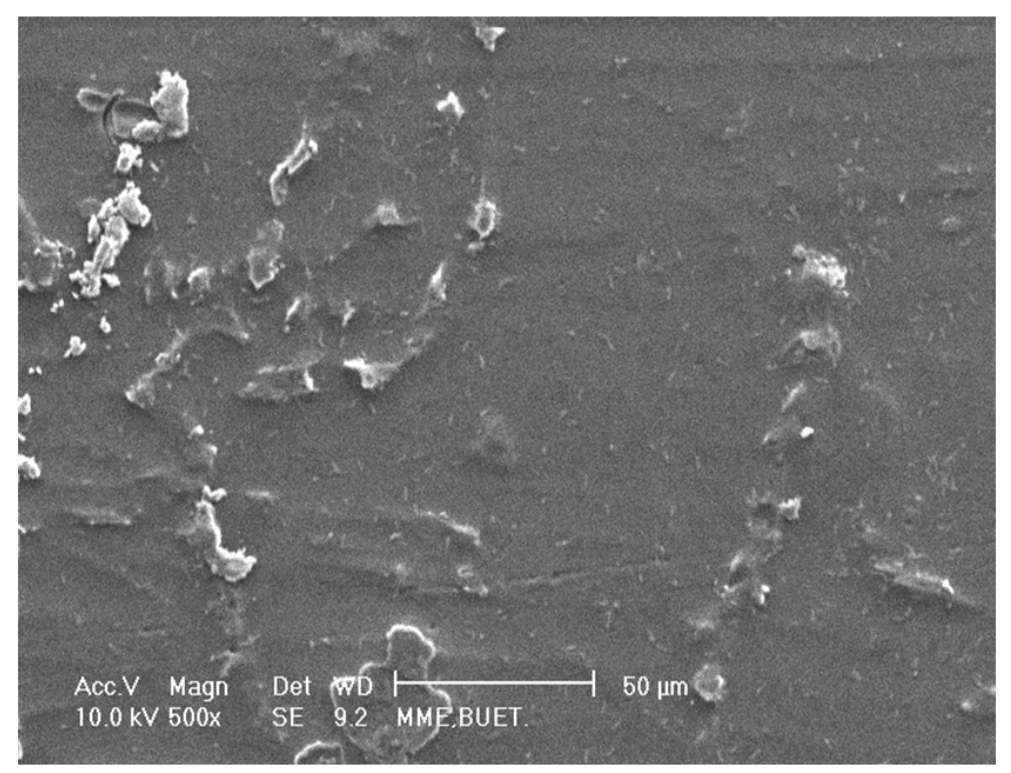

Figure 11. SEM image of gamma untreated pure PVA film.

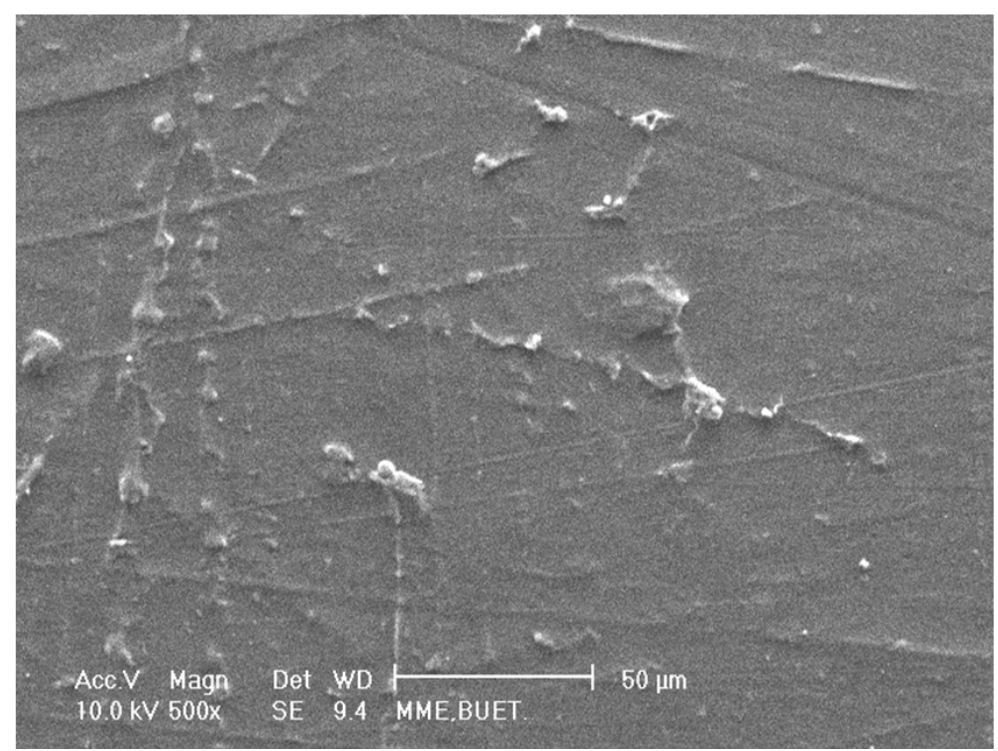

Figure 12. SEM image of gamma irradiated P2 formulation (95\% PVA + 5\% gelatin).

control sample. The highest Eb (165\%) was found for P3 formulation (90\% PVA + 10\% gelatin) at 100 krad dose. Finally, it can be concluded that irradiation increased the mechanical properties of the PVA and gelatinbased films.

\section{Acknowledgements}

Authors would like to thanks to the staff of the Institute of Radiation and Polymer Technology, Bangladesh Atomic Energy Commission.

\section{References}

[1] Srinivaasa, P.C., Ramesh, M.N., Kumar, K.R. and Tharanathan, R.N. (2003) Properties and Sorption Studies of Chitosan-Polyvinyl Alcohol Blend Films. Carbohydrate Polymers, 53, 431-438. 
http://dx.doi.org/10.1016/S0144-8617(03)00105-X

[2] Lee, Y.J., Shin, D.S., Choi, H.G., Han, S.S. and Lyoo, W.S. (2007) Preparation of Atactic Poly(vinyl alcohol)/Sodium Alginate Blend Nanowebs by Electrospinning. Journal of Applied Polymer Science, 106, 1337-1342. http://dx.doi.org/10.1002/app.26568

[3] WHO (1979) Evaluation of the Carcinogenic Risk of Chemicals to Man. Vol. 19, IARC Working Group, Lyon, 341366.

[4] Gul-E-Noor, F., Khan, M.A., Ghoshal, S., Mazid, R.A., Chowdhury, S.A.M. and Khan, R.A. (2009) Grafting of 2Ethylhexyl Acrylate with Urea on to Gelatin Film by Gamma Radiation. Journal of Macromolecular Science, 46, 615624.

[5] Sarker, B., Dey, K. and Khan, R.A. (2011) Effect of Incorporation of Polypropylene on the Physico-Mechanical and Thermo-Mechanical Properties of Gelatin Fiber Based Linear Low Density Polyethylene Bio-foamed Composite. Journal of Thermoplastic Composite Materials, 24, 679-694.

[6] Khan, R.A., Sarker, B., Khan, A., Huq, T., Noor, N. and Khan, M.A. (2010) Fabrication and Characterization of Gelatin Fiber-based Linear Low-density Polyethylene Foamed Composite. Journal of Reinforced Plastics and Composites, 29, 2438-2449. http://dx.doi.org/10.1177/0731684409351168

[7] Zaman, H.U., Khan, M.A. and Khan, R.A. (2013) Comparison of Mechanical and Degradation Properties of EG and EGDMA Grafted Gelatin Films. Journal of Adhesion Science and Technology, 27, 413-422. http://dx.doi.org/10.1080/01694243.2012.707450

[8] Zaman, H.U., Beg, M.D.H., Khan, M.A. and Khan, R.A. (2013) A Comparative Study of Gamma and Ultraviolet Radiation on gelatin Film with 2-Ethylhexyl Acrylate. Journal of Adhesion Science and Technology, 27, 2653-2665.

[9] Tharanatha, R.N. (2003) Biodegradable Films and Composite Coatings: Past, Present, and Future. Journal of Food Science and Technology, 14, 71-78.

[10] Khan, M.A. and Hasan, M.M. (2004) Polymer Surface Modification: Relevance to Adhesion, VSP. Vol. 3, CRC Press, Boca Raton, 263-283. http://dx.doi.org/10.1201/b12183-16

[11] Khan, M.A., Khan, R.A., Noor, F.G., Rahman, M.M. and Noor-A-Alam, M. (2009) Studies on the Mechanical Properties of Gelatinand Its Blends with Vinyltrimethoxysilane: Effect of Gamma Radiation. Polymer-Plastics Technology and Engineering, 48, 813-818.

[12] Zaman, H.U., Khan, A.H., Hossain, M.A., Khan, M.A. and Khan, R.A. (2009) Mechanical and Electrical Properties of Jute Fabrics Reinforced Polyethylene/Polypropylene Composites: Role of Gamma Radiation. Polymer-Plastics Technology and Engineering, 48, 760-766. http://dx.doi.org/10.1080/03602550902824655

[13] Czvikovszky, T. (1995) Reactive Recycling of Multiphase Polymer Systems through Electron Beam. Nuclear Instruments and Methods in Physics Research Section B, 105, 233-237. http://dx.doi.org/10.1016/0168-583X(95)00528-5

[14] Ressouany, M., Vachon, C. and Lacroix, M. (1998) Irradiation Dose and Calcium Effect on the Mechanical Properties of Cross-Linked Caseinate Films. Journal of Agricultural and Food Chemistry, 46, 1618-1623. http://dx.doi.org/10.1021/jf970805z

[15] Jo, C., Kang, H., Lee, N.Y., Kwon, J.H. and Byun, M.W. (2005) Pectin- and Gelatin-Based Film: Effect of Gamma Irradiation on the Mechanical Properties and Biodegradation. Radiation Physics and Chemistry, 72, 745-750.

[16] Park, H.J., Rhim, J.H., Jung, S.T. and Kang, S.G. (1995) Mechanical Properties of Carrageenan-Based Biopolymer Films. Journal of KOPAST, 1, 38-50.

[17] Peppas, N.A. (1986) Hydrogels in Medicine and Pharmacy. Vol. 1, CRC Press Inc., Boca Raton, 1.

[18] Song, C.L., Yoshii, F. and Kume, T.J. (2001) Radiation Crosslinking of Biodegradable Poly(butylene succinate) at High Temperature. Macromol. Sci., Pure and Applied Chemistry Part A, 38, 961-971.

[19] Khan, F. and Ahmad, S.R. (1997) Graft Copolymerization Reaction of Water-Emulsified Methyl Methacrylate with Preirradiated Jute Fiber. Journal of Applied Polymer Science, 65, 459-468. http://dx.doi.org/10.1002/(SICI)1097-4628(19970718)65:3<459::AID-APP5>3.0.CO;2-G

[20] Hsu, S.C., Don, T.M. and Chiu, W.Y. (2002) Synthesis of Chitosan-Modified Poly(methyl methacrylate) by Emulsion Polymerization. Journal of Applied Polymer Science, 86, 3047-3056. http://dx.doi.org/10.1002/app.11333 